\title{
C̆erenkov Line Radiation: An Important Line Mechanism in the Broad-Line Region ${ }^{1}$
}

Jian-rong Shi, Hao Wu, and Jun-han You

Institute for Space and Astrophysics, Department of Applied Physics, Shanghai Jiaotong University, Shanghai 200030, China

\section{Introduction}

Observations (Espey et al. 1989, Carswell et al. 1991, Marziani et al. 1996) at high resolution have shown relative shifts between the Balmer lines and the CIV line in a number of QSOs. In this paper, we suggest that in the BLR there are many cloudlets with dense gas that is optically thick, and the BLR is not only illuminated by UV-X radiation, but also by relativistic electrons. Therefore the Čerenkov line radiation has to be taken into consideration, and we calculate the Čerenkov line redshifts and the profiles.

\section{The Redshifts and Profiles of Čerenkov Lines}

The basic formula for calculating the Čerenkov line redshift $U_{t}$ is

$$
\Delta z^{c} \equiv U_{t} \equiv \frac{\Delta \lambda_{t}}{\lambda_{l u}}=\frac{1}{C_{0}^{-1} \gamma_{c}^{-2}+\sqrt{C_{0}^{-2} \gamma_{c}^{-4}+\left(C_{3} / C_{2}\right)}} .
$$

Here $\gamma_{c}$ is the characteristic electron energy in a given source, and the constants $C_{0}, C_{2}$, and $C_{3}$ are given by You \& Cheng (1986). For the very dense gas (say, $N_{\mathrm{H}} \approx 10^{16} \mathrm{~cm}^{-3}$ ) that we are interested in, eq. (1) can be simplified as

$$
\Delta z_{c} \equiv U_{t} \simeq \sqrt{\frac{C_{2}}{C_{3}}}=1.04 \times 10^{-11} \sqrt{\lambda_{l u} A_{u l} \Gamma_{l u}\left(\frac{g_{u}}{g_{l}}\right) R_{l} R_{p}^{-1} p^{5} \xi},
$$

where $\xi=N / N_{\mathrm{H}}^{0}$ is the number density ratio of relevant atomic (ion) species to neutral hydrogen, $N_{\mathrm{H}}$ is the number density of hydrogen atoms, $N$ is the number density of the relevant atom (ion), $g_{u}$ and $g_{l}$ are the degeneracy of the upper and lower levels respectively, $R_{l} \equiv N_{l} / N$ denotes the fractional population of the lower level $l$ of relevant atom (ion), and $R_{p} \equiv N_{\mathrm{H} p}^{0} / N_{\mathrm{H}}^{0}$ is the fractional population at the lowest photoionization level $p$ of the hydrogen atom.

As an example, we calculate the redshifts of the Čerenkov $\mathrm{H} \beta$ line, for which $A_{42}=3.33 \times 10^{7} \mathrm{sec}^{-1}, \lambda_{24}=4.861 \times 10^{-5} \mathrm{~cm}, \Gamma_{24}=7.44 \times 10^{8} \mathrm{sec}^{-1}, p=3$, taking $R_{2} \simeq 10^{-2}$ (the population in level 2 is anomalously increased due to Ly $\alpha$ trapping), and taking $T=1.5 \times 10^{4} \mathrm{~K}$. Taking the density of hydrogen gas $N$ as

\footnotetext{
${ }^{1}$ This work is supported by National Natural Science Foundation of China.
} 

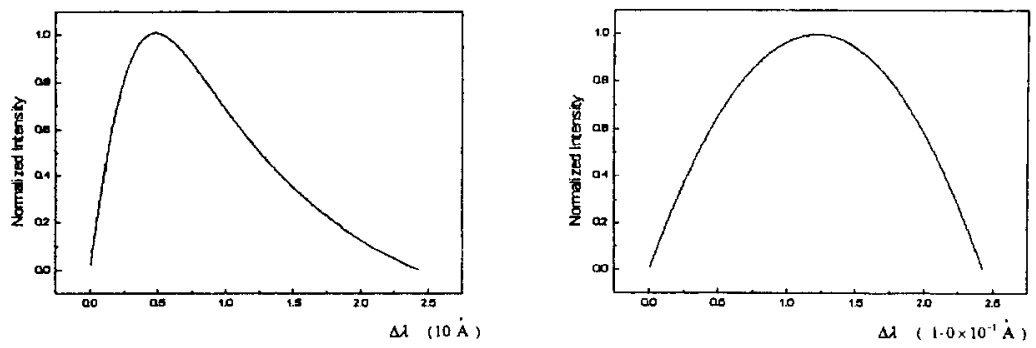

Figure 1. The Čerenkov line $\mathrm{H} \beta$ profiles at $T=1.5 \times 10^{4} \mathrm{~K}$. Left: $N=10^{16} \mathrm{~cm}^{-3}$. Right: $N=10^{14} \mathrm{~cm}^{-3}$.

a free parameter, the calculated the redshifts of Čerenkov $\mathrm{H} \beta$ line are shown in Table 1. The profiles are shown in Fig. 1.

Table 1. $\breve{C}$ erenkov Line $\mathrm{H} \beta$ Redshifts

\begin{tabular}{cc}
\hline \hline Density $N\left(\mathrm{~cm}^{-3}\right)$ & $\Delta z^{c}$ \\
\hline $10^{16}$ & $9.55 \times 10^{-4}$ \\
$10^{14}$ & $2.50 \times 10^{-5}$ \\
$10^{12}$ & $2.50 \times 10^{-7}$
\end{tabular}

\section{Conclusions and Discussion}

From eq. (2) we see that $\Delta z^{c}$ is dependent on $R_{l}$ and $R_{p}$, the particle population at different energy levels. Therefore $\Delta z^{c}$ is related to the temperature. The calculated $\Delta z^{c}(\mathrm{H} \beta)$ is about $10^{-3}$ (for very dense gas), which is in agreement with the observations. According to eq. (1), the different C̆erenkov lines have different redshifts, even for the lines of the same atomic species. So it is not difficult to understand the redshift difference between the $\mathrm{H} \alpha$ and $\mathrm{H} \beta$ lines, etc.

\section{References}

Carswell, R.F., et al. 1991, ApJ, 381, L5.

Espey, B. R., et al. 1989, ApJ, 342, 666.

Marizini, P., et al. 1996, ApJS, 104, 37.

You. J. H., \& Cheng, F. H. 1986, Phys. Rev. A, 34, 3015. 